\title{
Wavelet analysis of instrumented rocket motor data
}

D. H. Smith*

(Received 8 August 2003; revised 12 March 2004)

\begin{abstract}
Second generation wavelets by the lifting scheme are applied to irregularly sampled data from instrumented rocket motors and compared with a complementary discrete Fourier approach. Particular attention is given to ill-conditioning induced by mesh irregularity.
\end{abstract}

\section{Contents}

1 Introduction

2 DWT's and DFT's on irregular meshes

2.1 Second generation wavelets by the lifting scheme . . . . . C108

2.2 A discrete Fourier transform . . . . . . . . . . . . . . C110

${ }^{*}$ DSTO Edinburgh, P.O. Box 1500, Edinburgh, South Australia 5111. mailto:david.h.smith@dsto.defence.gov.au

See http://anziamj.austms.org.au/V45/CTAC2003/Smit/home.html for this article, (c) Austral. Mathematical Soc. 2004. Published March 26, 2004. ISSN 1446-8735 
3 Application to data

C110

3.1 Wavelet and Fourier representations . . . . . . . . . . C111

3.2 Detection of thermal measurement oscillation . . . . . . C C113

3.3 Ill-conditioning induced by mesh irregularity . . . . . . . C C113

3.4 Noise reduction by coefficient thresholding . . . . . . . . C117

4 Summary and conclusions

C118

References

C119

\section{Introduction}

Transforming data to an alternative domain is often useful in revealing features not immediately apparent in the original measurements. The ubiquitous discrete Fourier transform [6] involves a complete switch to the frequency domain, providing frequency content and forfeiting evolution information. Wavelet transforms retain this information, providing a multi-scale decomposition which simultaneously captures evolution behaviour across a range of scales. This study is concerned with the application of discrete Fourier and wavelet transforms to irregularly sampled data from an exploratory data analysis perspective, with some emphasis given to properties and behaviour of the wavelet systems. Comparisons are drawn between the two different representations and the wavelet noise reduction facility is demonstrated. It is also shown that severe mesh irregularity has the potential to destabilise a wavelet basis, an ailment which is easily curable by an SVD approach.

\section{DWT's and DFT's on irregular meshes}

For discrete data on irregular meshes, standard analysis methods like discrete Fourier or first generation wavelet transforms are not immediately applicable. 
A simple option is to interpolate the data onto an equally spaced mesh and then apply these methods, however this incurs extra computational expense and can raise more issues than it solves, such as choice of interpolation scheme and the introduction of artefacts. For these reasons the transforms under consideration will be constructed on the original irregular meshes.

\subsection{Second generation wavelets by the lifting scheme}

Second generation wavelets constructed by the lifting scheme [8] reside on finite intervals and break free from the constraints inherent to their first generation counterparts, such as restriction to regular meshes. The lifting scheme [8] factorises a discrete wavelet transform into a sequence of lifting steps [3], each playing a specific role in shaping the wavelets and scaling functions that constitute the resulting multi-resolution analysis framework.

Discrete data $\left(t_{i}, y_{i}\right)$ is considered to reside on the top level of a mesh hierarchy, with level $j$ comprising $2^{j}$ intervals. The first lifting step, or lazy wavelet, is a down-sampling that transfers values onto the next coarse grid

$$
y_{j-1, k}=y_{j, 2 k}, \quad \gamma_{j-1, k}=y_{j, 2 k+1} .
$$

Subsequent dual lifting is a high pass filter involving prediction of the odd indexed values from the fine level to yield wavelet coefficients representing the deviation between fine and coarse level approximations

$$
\gamma_{j-1, k}=\gamma_{j-1, k}-P\left(\ldots, y_{j-1, k}, y_{j-1, k+1}, \ldots\right),
$$

or loss in detail. Primal lifting is a low pass filter responsible for maintaining a lower resolution version of the data via an update

$$
y_{j-1, k}=y_{j-1, k}+U\left(\ldots, \gamma_{j-1, k-1}, \gamma_{j-1, k}, \ldots\right),
$$

where the update operator $U$ is chosen to impart one or more vanishing moments on the primal wavelet functions. Additional lifting steps may then be performed to produce desired properties on the MRA [8]. 

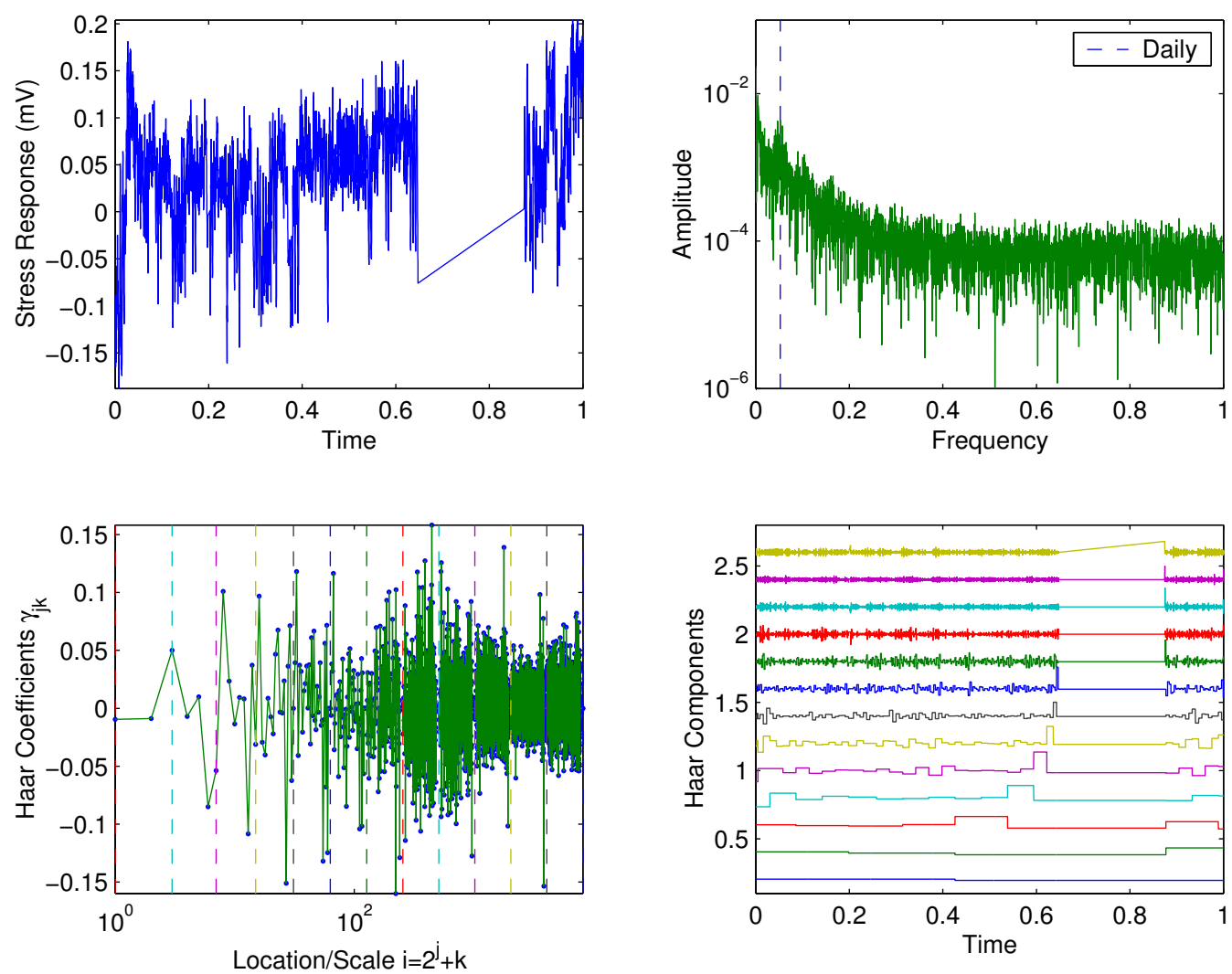

FIGURE 1: Irregularly sampled stress response data, comprising 7270 points, in the time, frequency and Haar wavelet domains. 


\subsection{A discrete Fourier transform}

A DFT for irregular temporal sampling may be defined by mapping the data to $[0,2 \pi)$ and considering trigonometric interpolation [1]

$$
y_{j}=\sum_{k=-(N+r) / 2}^{(N-r) / 2} \alpha_{k} e^{i k t_{j}}, \quad j=0, \ldots, N \quad(r=\operatorname{rem}(N, 2)),
$$

which reduces to the usual DFT in the regularly sampled case. These equations represent an inverse transform to recover the data from its Fourier coefficients, and the required task is accurate approximation of the coefficients given $\left(t_{j}, y_{j}\right)$. To avoid construction of the matrix $A_{j k}=e^{i k t_{j}}$, the right hand side of (1) is approximated by truncated Taylor series expansions about the nearest neighbours of the irregular nodes $t_{j}$, from a regular grid, with all function evaluations and derivatives rapidly computed by the FFT. The solution of (1) is then sought by Generalised Minimal Residual (GMRES) iteration preconditioned with a normal FFT, defining the forward transform. On highly irregular grids with large disparity in time steps, the equally spaced frequencies in (1) may be poorly matched to the actual frequencies present in the data, with the highest value not sufficiently large, leading to ill-conditioning and slow convergence.

\section{Application to data}

Figure 1 plots stress response data from a sensor inside a rocket motor subjected to ambient thermal loading conditions over 190 days [5]. Apart from the obvious large gap, mesh irregularity is present across the entire interval. Both discrete transforms will now be applied to compare some of their properties on this data set, including the issue of scale-frequency parity. 


\subsection{Wavelet and Fourier representations}

A discrete Haar wavelet transform, with predict and update

$$
P=y_{j-1, k}, \quad U=\frac{\Delta_{j, 2 k+1}}{\Delta_{j-1, k}} \gamma_{j-1, k}, \quad\left(\Delta_{j k}=t_{j, k+1}-t_{j k}, \quad j \leq n\right),
$$

produces a spectrum which can be viewed in several ways. One is to simply plot the coefficients $\gamma_{j k}$ at their respective scales and locations, giving the diagram in Figure 1 where each vertical band coincides with a resolution level and houses its $2^{j}$ coefficients. To see the actual evolution occurring on each level, wavelet components are defined by the summation

$$
\psi_{j}(t)=\sum_{k=0}^{2^{j}-1} \gamma_{j k} \psi_{j k}(t), \quad j=0, \ldots, n-1,
$$

and obtained by repeated inversion with all coefficients set to zero except those on level $j$. A notable feature of this plot is the locally large values emanating from both sides of the data gap, an effective singularity, which is connected with the singularity detection properties of wavelets. Accompanying the wavelet spectrum is a DFT amplitude spectrum with five Taylor series terms, produced after GMRES stagnation at relative residual 0.086. In this representation the time axis is absent and a peak is present at daily frequency, which lies near levels 7-8 in the wavelet domain, where a clear bulge is visible in the coefficient envelope. Figure 2 illustrates this scale-frequency parity by giving truncated wavelet and Fourier expansions with components above daily resolution removed, also demonstrating the lack of smoothness inherent to Haar wavelets. Reconstruction errors for truncated wavelet expansions are also shown alongside equivalent Fourier truncations, indicating similar behaviour with respect to the level of compression. The distinct elbow in these curves is related to a transition between dominance of fine scale noise-like features and their larger scale counterparts in the reconstruction.

While the DFT identifies daily frequency components which are not as readily apparent in the Haar spectrum, it does so at enormous computational 

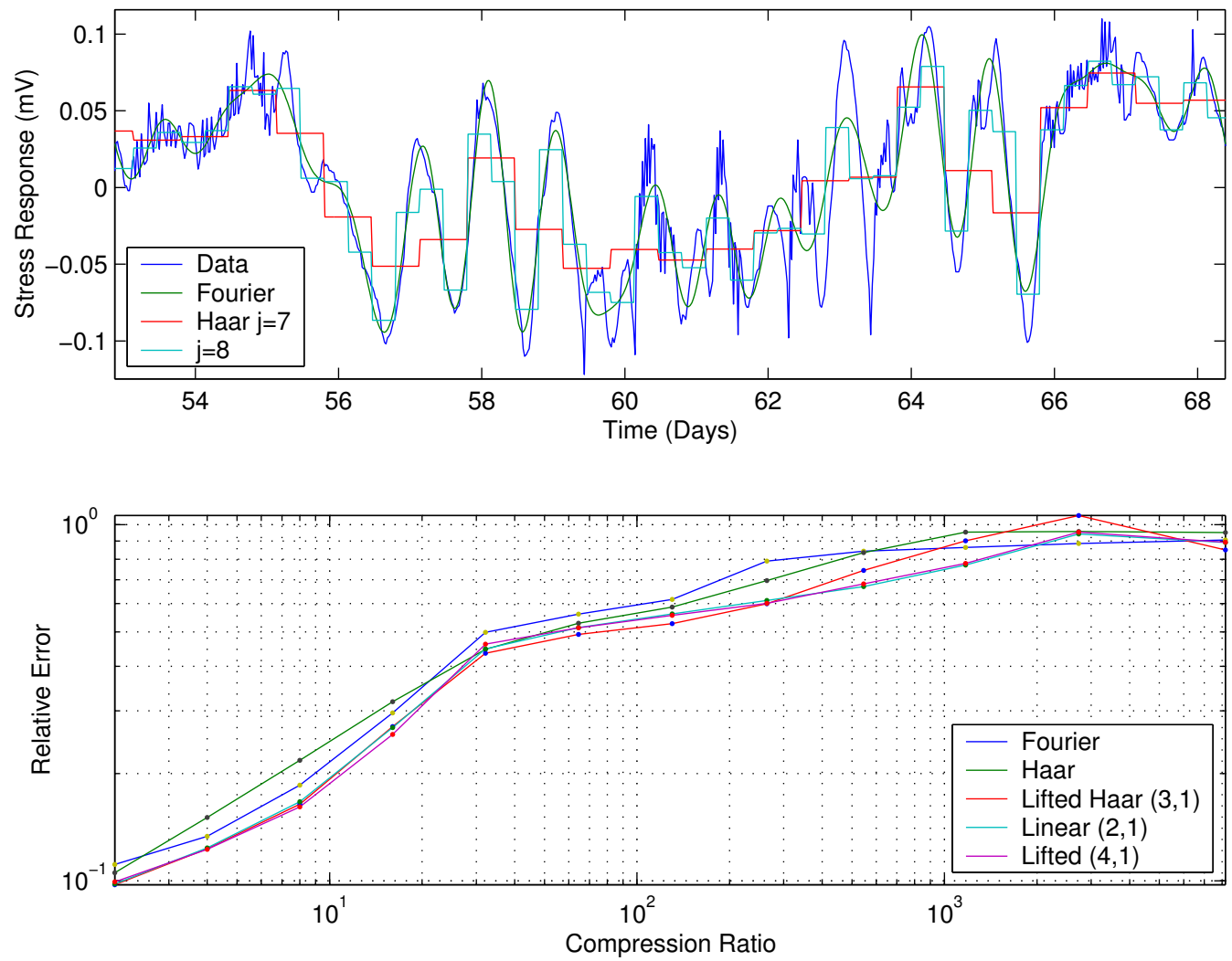

Figure 2: Truncated wavelet and Fourier expansions of the data in Figure 1, showing daily reconstructions and error behaviour for several wavelets. 
cost relative to the DWT, and loses evolution information in the process. The cost disparity is largely due to poor GMRES convergence and could be reduced by a better preconditioner, possibly employing wavelets.

\subsection{Detection of thermal measurement oscillation}

A second data set comprising 22979 stress response measurements under a more aggressive thermal cycling between $\pm 50^{\circ}$ over approximately 300 days is shown in Figure 3, displaying typical viscoelastic relaxation during the cold cycle portions. Oscillatory behaviour, which clearly displays different amplitude characteristics during the hot, cold and shorter transition periods, is elucidated by considering wavelet components. The figure includes Haar and linear $(2,1)$ components, constructed by a linear predict operator, on the finest scale $(j=14)$ and a coarser scale $(j=10)$, during the fourth cold cycle and its neighbouring transition regions. Perhaps the most striking feature is an abrupt vanishing of the finest scale components outside the cold region, suggesting a certain thermal dependence of the measuring device. Daily components represented on the coarser scale $j=10$ display some local irregularity in the transition neighbourhood due to suspected non-physical data spikes, and persist into the transition unlike their fine scale counterparts.

\subsection{Ill-conditioning induced by mesh irregularity}

Application of dual lifting to the linear $(2,1)$ wavelet system yields new coefficients according to a second predict operation performed after the update

$$
\gamma_{j-1, k}=\gamma_{j-1, k}+\sum_{l=-1}^{2} \theta_{j-1, k+l}^{k} y_{j-1, k+l},
$$

which draws on additional neighbours and generates new primal scaling functions, primal wavelets and dual wavelets, while leaving the dual scaling func- 

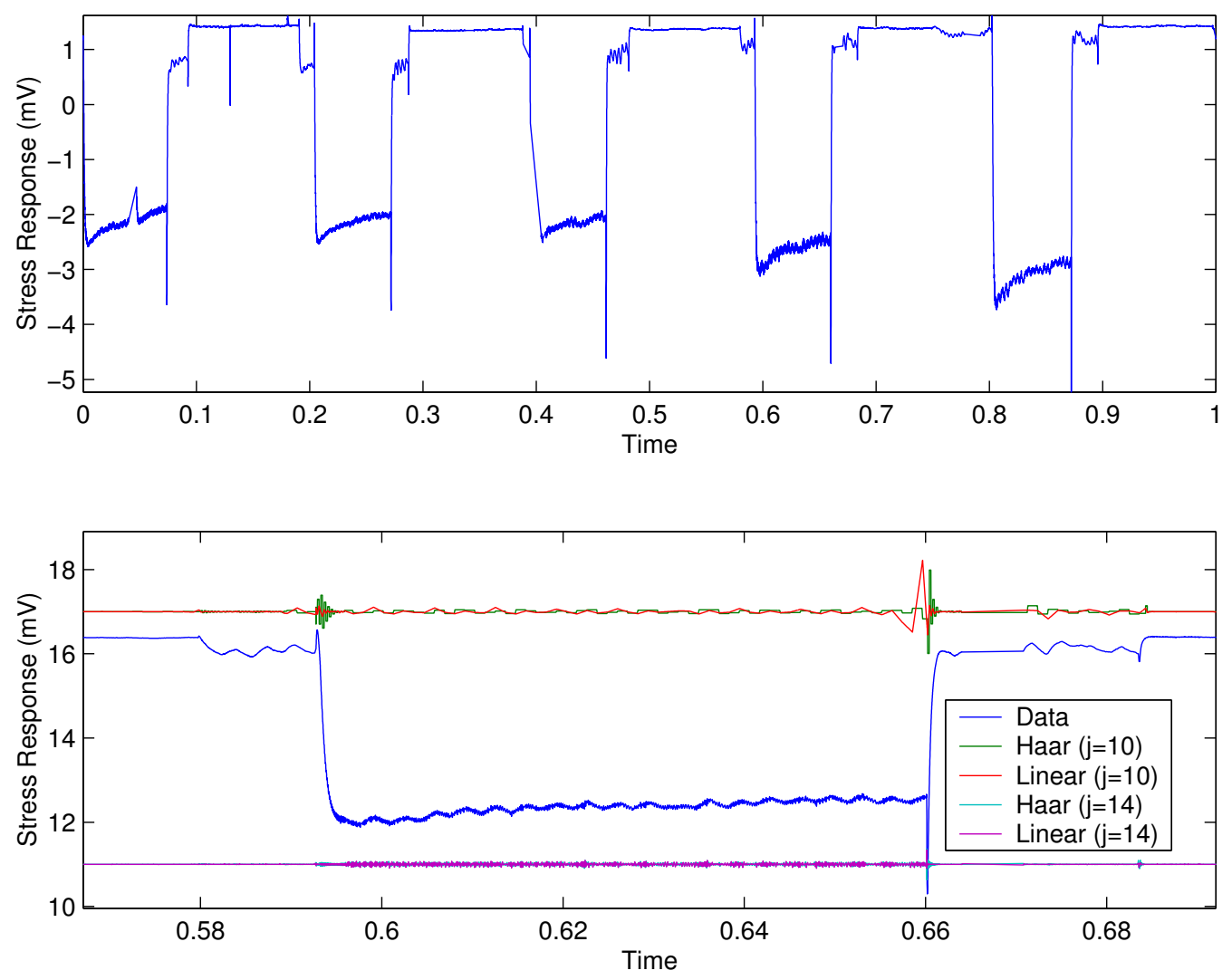

Figure 3: Thermal cycling data with Haar and linear $(2,1)$ wavelet components on levels 14(top) and 10(daily). 
tions unchanged [8]. A refinement relation expresses the dual wavelets in terms of dual scaling functions on adjacent resolution levels

$$
\widetilde{\psi}_{j-1, k}=-\beta_{j-1, k} \widetilde{\varphi}_{j, 2 k}+\widetilde{\varphi}_{j, 2 k+1}+\left(1-\beta_{j-1, k}\right) \widetilde{\varphi}_{j, 2 k+2}+\sum_{l=-1}^{2} \theta_{j-1, k+l}^{k} \widetilde{\varphi}_{j-1, k+l},
$$

from which the necessary integration is performed to enforce four vanishing moments, producing a linear system of equations to be solved on each coarse grid cell for the dual lifting coefficients $\theta_{j-1, k+l}^{k}$. The exact solution is expressed in terms of neighbouring cell widths on adjacent resolution levels, reducing to $\pm \frac{1}{16}$ on regular grids, with symmetric wavelets.

Application of (2) to the data set in Figure 1 confirms an expected smoothing effect on the level components, however the same treatment on the data of Figure 3 produces an unexpected outcome, in which level components contain spikes as high as $10^{7}$. Inversion still recovers the original data, however to achieve this means precarious cancellation is taking place between excessive wavelet components on different levels, in much the same way as for ill-conditioned least squares data fitting [6]. The damage is caused here by a pathological case of grid irregularity, with sudden large changes in grid spacing generating dual lifting coefficients exceeding one in magnitude, causing divergence of the subdivision scheme responsible for the wavelets and scaling functions. This ill-conditioning of the wavelet basis has also been observed by Simoens and Vandewalle in the context of biorthogonal wavelet systems constructed by average interpolating subdivision [7], and cured by the application of constrained local semi-orthogonalisation, which offers stabilisation with preserved vanishing moments at the expense of altering the wavelet support. An alternative approach which maintains the wavelet support while compromising vanishing moments involves truncated SVD solutions, in which large solution components along near null space vectors with small singular values are removed [6]. Table 1 provides details of this process at one of only 7 grid locations in which instability arises during the first transform step, showing typical hallmarks of ill conditioning in the 

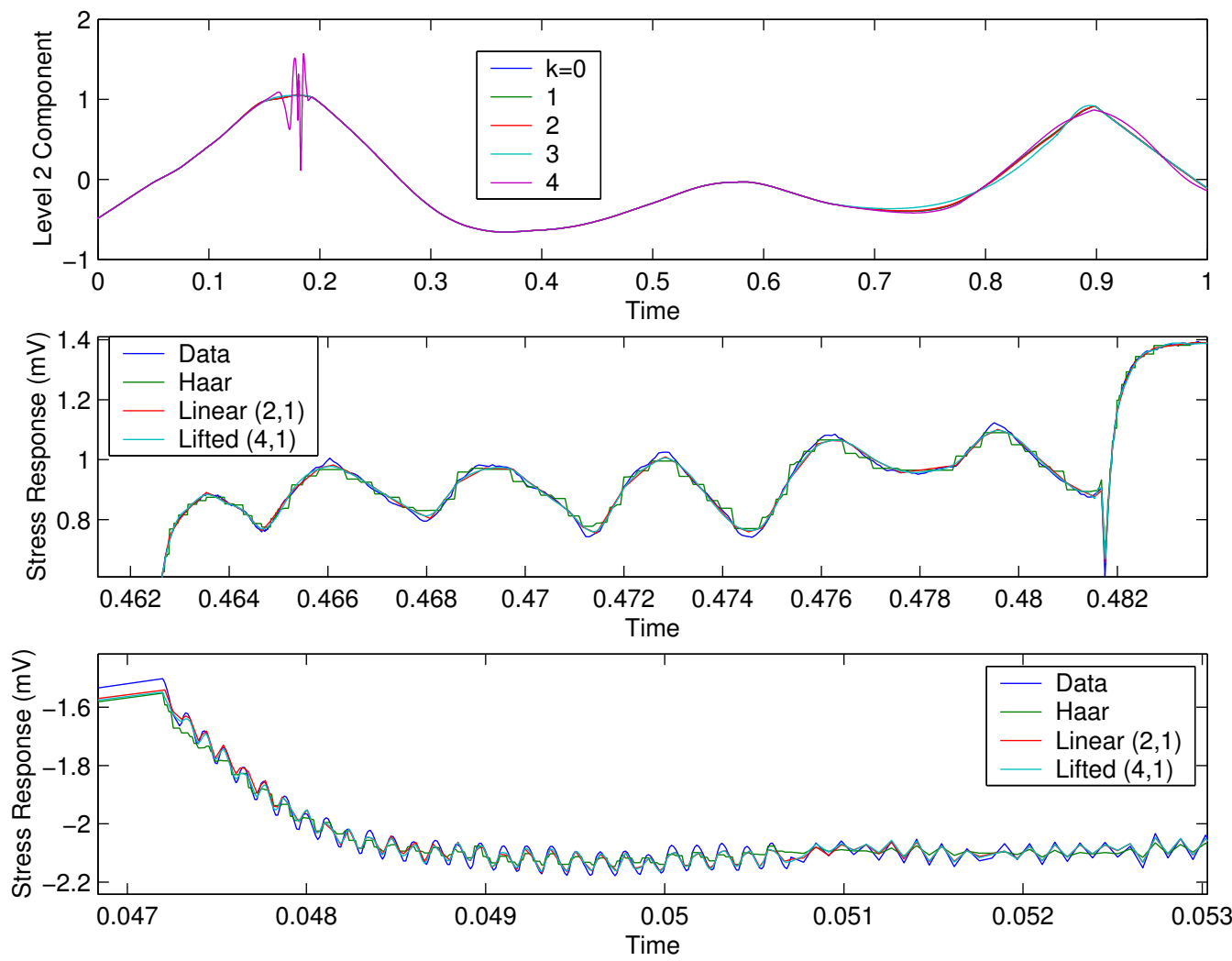

Figure 4: Cure of ill-conditioned $(4,1)$ wavelet basis by SVD truncation, accompanied by close up views of soft thresholding on the data of Figure 3. 
TABLE 1: Local ill conditioning caused by severe grid irregularity during the first step of a $(4,1)$ wavelet transform applied to the data in Figure 3.

\begin{tabular}{|c|ccccc|}
\hline & Exact Formula & SVD $k=1$ & $k=2$ & $k=3$ & $k=4$ \\
\hline$\|\theta\|$ & 95.1242 & $7.8210 \mathrm{e}-9$ & $4.3517 \mathrm{e}-5$ & 0.2928 & 0.5593 \\
$\|A \theta-b\|$ & 15.74 & 0.9882 & 0.2056 & 780.7 & 784.5 \\
\hline
\end{tabular}

computed residual behaviour. Cure of the wavelet components on level 2 is shown in Figure 4, displaying the regularisation process as $k$ varies.

\subsection{Noise reduction by coefficient thresholding}

Thresholding is the selective removal or reduction of wavelet coefficients with the aim of producing a "cleaned" signal upon inversion. The implication is that the signal is essentially composed from a relatively small number of "large" coefficients, while "small" coefficients are attributed to noise and can be discarded. Hard thresholding replaces such coefficients with zero while soft thresholding also shrinks the remaining coefficients by an amount equal to the deviation between threshold and coefficient. Consider the data as values of a function contaminated by zero mean additive noise

$$
y_{j}=f\left(t_{j}\right)+\varepsilon_{j},
$$

and apply a wavelet transform via the sparse matrix $W$. The resulting computed wavelet coefficients will then be unbiased estimates of their exact counterparts, and for uncorrelated noise of variance $\sigma^{2}$ the covariance matrix is

$$
V=E\left[(W \varepsilon)(W \varepsilon)^{T}\right]=\sigma^{2} W W^{T},
$$

from which individual coefficient variance appears on the diagonal. The matrix $W W^{T}$, dependent only on the particular wavelet system and temporal grid, emerges as a key indicator of noise structure in the wavelet domain, providing the mechanism for noise distribution among resolution levels. Correlation dependent thresholding [2], designed for biorthogonal wavelet systems, 
applies a modified universal threshold to coefficient $\gamma_{j k}$

$$
\delta_{j k}=\hat{\sigma}_{j k} \sqrt{2(1+\zeta) \log M},
$$

where $\hat{\sigma}_{j k}$ is a standard deviation estimate for $\gamma_{j k}, \zeta$ is a bound on the cross correlation between wavelet coefficients on different levels and $M$ is the total number of wavelet coefficients. Normalising the computed wavelet coefficients by their variance and applying a median absolute deviation estimate gives $\hat{\sigma}_{j k}$ without prior knowledge of the noise variance $\sigma^{2}[4]$.

Figure 4 shows soft thresholding results on the data of Figure 3 by three wavelet systems, with Haar clearly showing its smoothness disadvantage. In each case thresholding is performed on coefficients from the top half of resolution levels. Suspected non-physical large spikes, or outliers, are essentially untouched by the process, which only acts against small quantities.

\section{Summary and conclusions}

Biorthogonal wavelet systems built by the lifting scheme were applied to irregularly sampled data from thermally loaded rocket motors, with comparisons drawn against a complementary discrete Fourier transform. Poor convergence of the iterative DFT algorithm due to inadequate preconditioning limited its application despite successful identification of daily components, while similar data reconstruction error behaviour was observed for truncated and Fourier expansions. In a different data set the mathematical microscope property of wavelets was demonstrated in separating thermal oscillatory behaviour associated with the measuring device. A case of pathological grid irregularity uncovered instability in the dual lifted $(4,1)$ wavelet system, which was cured by the application of SVD methods, however a precise understanding of this in terms of biorthogonality needs further study. Limited noise reduction results with several wavelets pointed against the use of Haar while confirming the need for additional outlier procedures. 


\section{References}

[1] C. Anderson, M. Dahleh, "Rapid Computation of the Discrete Fourier Transform", SIAM J. Sci. Stat. Comp. 174 p913-919 (1996). C110

[2] K. Berkner and R.O. Wells Jr., "A Correlation-Dependent Model for Denoising via Nonorthogonal Wavelet Transforms", Rice University Technical Report CML9807, (1998). C117

[3] I. Daubechies, W. Sweldens, "Factoring Wavelet Transforms into Lifting Steps", J. Fourier Analysis and Appl. 43 p247-269 (1998). C108

[4] V. Delouille, J. Simoens and R. von Sachs, "Smooth Design-adapted Wavelets for Nonparametric Stochastic Regression", Discussion paper 0117, Institut de Statistique, Universit Catholique de Louvain, (2001). C118

[5] P. Macdowell, "New Service Life Methodologies for Solid Propellant Rocket Motors", DSTO-RR-0099 (1999). C110

[6] Numerical Recipes, http://www.nr.com. C107, C115

[7] J. Simoens and S. Vandewalle, "A Stabilised Lifting Construction of Wavelets on Irregular Meshes on the Interval", SIAM J. Sci Computing 244 p1356-1378 (2003). C115

[8] W. Sweldens, "The Lifting Scheme: A Construction of Second Generation Wavelets", SIAM J. Math. Analysis 292 p511-546 (1998). C108, C115 